\title{
Psychological outcomes after hospitalization for COVID-19: data from a multidisciplinary follow-up screening program for recovered patients
}

\author{
Federica Bonazza,${ }^{1 *}$ Lidia Borghi, ${ }^{1 *}$ Eugenia Cao di San Marco, ${ }^{2}$ Kyrie Piscopo, ${ }^{2}$ Francesca Bai, ${ }^{3}$ \\ Antonella d'Arminio Monforte, ${ }^{3}$ Elena Vegni ${ }^{1,2}$
}

${ }^{1}$ Department of Health Science, University of Milan; ${ }^{2}$ Unit of Clinical Psychology, Santi Paolo and Carlo Hospital, Milan; ${ }^{3}$ Clinic of Infectious Diseases and Tropical Medicine, Department of Health Sciences, University of Milan, ASST Santi Paolo e Carlo, Milan, Italy

Correspondence: Federica Bonazza, Department of Health Science, University of Milan, via A. di Rudinì 8, 20142 Milan, Italy.

E-mail: federica.bonazza@guest.unimi.it

Citation: Bonazza, F., Borghi, L., Cao di San Marco, E., Piscopo, K., Bai, F., d'Arminio Monforte, A., \& Vegni E. (2020). Psychological outcomes after hospitalization for COVID-19: data from a multidisciplinary follow-up screening program for recovered patients. Research in Psychotherapy: Psychopathology, Process and Outcome, 23(3), 247-255. doi: 10.4081/ripppo.2020.491

Acknowledgments: the authors would like to thank Chiara Luridiana, who helped in collecting data.

Contributions: FB and LB are co-first authors. FeB, LB and EC have written the first draft of the study. FeB and FrB have collected data and LB has performed the data analysis. FeB, LB, EC, KP, $\mathrm{FrB}, \mathrm{AdM}, \mathrm{EV}$ critically revised the manuscript and approved the final version.

Conflict of interest: the authors declare no potential conflict of interest.

Ethical approval and consent to participate: the study was conducted in accordance with the Declaration of Helsinki Ethical Principles and the broader longitudinal research which comprehends the present study has been approved by the Ethical Committee of the University of Milan.

Consent for publication: the present study has been approved by the Ethical Committee of the University of Milan. Participants gave consent for publication.

Availability of data and material: the data that support the findings of this study are available from the corresponding author $[\mathrm{FeB}]$ on request.

Received for publication: 2 September 2020.

Revision received: 21 October 2020.

Accepted for publication: 4 November 2020.

This work is licensed under a Creative Commons Attribution NonCommercial 4.0 License (CC BY-NC 4.0).

${ }^{\circ}$ Copyright: the Author(s), 2020

Licensee PAGEPress, Italy

Research in Psychotherapy:

Psychopathology, Process and Outcome 2020; 23:247-255

doi:10.4081/ripppo.2020.491

\begin{abstract}
Patients who are hospitalized for coronavirus disease 2019 (COVID-19) face an extremely stressful experience that might challenge their mental health. The study aims to describe the psychological condition of recovered patients, focusing on anxiety and depression symptoms, as well as post-traumatic stress. All the recovered COVID-19 patients who accessed to a multidisciplinary follow-up screening program scheduled within two months after their hospital discharge were included. As far as the psychological assessment, patients completed the Hospital Anxiety and Depression Scale and the Impact of Event ScaleRevised for post-traumatic stress. Socio-demographic and clinical data (days of hospitalization, intensity of received care, and number of supportive sessions with the hospital psychologist after the hospitalization) were collected. Descriptive, correlation and regression analyses were conducted. The sample includes 261 patients $(68.2 \%$ men), aged between 23 and 90 (mean=58.9 st. dev=13.3). High numbers of patients reported anxiety $(28 \%)$ and depression symptoms (17\%), as well as post-traumatic stress (36.4\%). Impaired outcomes were associated with female gender, while patient's age was found to be negatively correlated with anxiety symptoms. $13.8 \%$ of patients underwent a psychological visit and $6.1 \%$ of them were taken in charge for psychological support. Few months after hospital discharge, individuals recovered by COVID-19 reported negative consequences on their mental health. Understanding the impact that COVID-19 and hospitalization have on recovered patients may provide insights about how to develop an effective psychological intervention to help them deal with such psychological distress and prevent further psychopathological effects.
\end{abstract}

Key words: Anxiety; depression; COVID-19 recovered patients; post-traumatic stress; mental health.

\section{Introduction}

The outbreak of the severe acute respiratory syndrome coronavirus 2 (SARS-CoV-2) originated in December 2019 in the Chinese province of Hubei and has rapidly spread worldwide, affecting 216 countries and 17,106,007 people and causing 668,910 deaths (World Health Organization

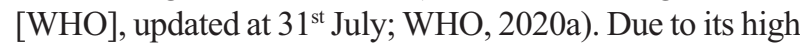
rate of transmissibility, hospitalization and mortality, on $11^{\text {th }}$ March 2020 the WHO defined this epidemic as a pandemic. 
Italy was among the first countries experiencing SARSCoV-2 outbreak, with a total number of 247,537 positive cases at $31^{\text {st }}$ July, the vast majority located in Lombardy region with 96,219 cases, 16,806 deaths and 73,402 recovered patients (Ministero della Salute, updated on $31^{\text {st July). }}$ During the first-wave of the pandemic in Italy, as in other countries, the implementation of the massive restrictive measurements (i.e. lockdown, stay-at-home orders, and physical distancing) was necessary to reduce the spread of the virus and to alleviate the pressure on the health care system (Remuzzi \& Remuzzi, 2020). Some studies have highlighted the psychological strain caused by the pandemic among the general population in Italy, reporting high or very high levels of distress, fear of being infected, anxiety and depression (Franceschini et al., 2020; Lenzo et al., 2020; Mazza et al., 2020; Rossi et al., 2020).

The infection may result in the COronaVIrus Disease 2019 (COVID-19), a severe systemic disease, in which pneumonia is the dominant clinical manifestation, but also other systems might be involved, such as cardiovascular, nephrological and neurological (Bansal, 2020; Bose \& McCarthy, 2020; Fiani, Covarrubias, Desai, Sekhon, \& Jarrah, 2020; Tadolini et al., 2020), leading to the need for hospitalization. Hospitalized COVID-19 patients had to deal with a complex and potentially highly stressful situation: as known from other infectious pandemics, patients have been exposed to several stressors such as isolation, uncertainty about treatment and prognosis, fear of death and of infecting loved ones, and lack of support from relatives (Chua et al., 2003). Moreover, Karademas, Tsagaraki and Lambrou (2009) conceptualized hospitalization as an adverse event, that puts a strain on patients' resources. Especially patients requiring hospitalization in Intensive Care Unit (ICU) experienced this event as traumatic, being exposed to invasive procedures and a drastic reduction in autonomy (Davydow, Gifford, Desai, Needham \& Bienvenu, 2008).

Thus, it is crucial to arrange a follow-up of recovered patients to early identify possible psychological distress and long-term sequelae (Balachandar et al., 2020). The prevalence of distress, anxiety and post-traumatic stress symptoms among COVID-19 patients was found to be significantly high (Bo et al., 2020), while, to date, data on follow-up of patients recovered from COVID-19 are scarce. Preliminary data suggest that anxiety and depression might also persist in patients recovering from COVID-19 (Rogers et al., 2020), and, in an Italian cohort, 30\% of patients showed anxiety or depressive symptoms at 1 to 3 months from recovery (Tomasoni et al., 2020).

Studies on previous infectious epidemics, like SARS, the Middle East respiratory syndrome (MERS) and the Ebola Virus Disease (EVD) outbreak, reported that psychological symptoms might persist or arise after the infection, with long-term negative outcomes (Troyer, Kohn \& Hong, 2020; Ahmed et al., 2020; Ji et al., 2017; Batawi et al., 2020). The SARS epidemic showed both a short-term and a long-term impact on mental health (Kwek et al., 2006), and among MERS survivors, patients who were admitted to ICU presented important limitations on routine activities because of emotional problems, and they were not able to handle changes caused by the disease (Batawi et al., 2020). In the same way, the 2014-2015 Ebola epidemic generated long-term physical and psychological sequelae in recovered patients, not only due to the disease itself, but also to the relatives' death, the lockdown, and the economic collapse (Ji et al., 2017); recovered individuals showed a high prevalence of distress, depression and anxiety symptoms (James, Wardle, Steel, \& Adams, 2020). Since the SARS-CoV-2 outbreak has parallels with the previous epidemics (SARS, MERS and Ebola) (Ahmed et al., 2020), there is a call for assessing and monitoring the psychological outcomes in the periods after the recovery, especially among hospitalized patients (Hong et al., 2009).

Psychological distress is therefore an important concern to be considered in patients with COVID-19 and should not be neglected during hospitalization and follow-up. The present contribution reports the cross-sectional data on a larger and longitudinal follow-up screening program aimed to assess the psychological short-term and long-term outcomes of recovered COVID-19 patients. In particular, within a multidisciplinary follow-up screening program offered to each patient who was hospitalized and recovered from COVID-19, data on anxiety, depression and post-traumatic stress were collected within 2 months from recovery, and will be assessed longitudinally, along with other posttraumatic dimensions.

In this context, we aimed to investigate the prevalence and possible socio-demographic or clinical predictors of anxiety, depression, and post-traumatic stress in recovered patients after hospitalization for COVID-19. In particular, male and older patients were expected to show a higher prevalence of distress, due to their higher exposure to severe forms of the disease and risk for death (Jin et al., 2020; Bhopal \& Bhopal, 2020). In addition, in line with the abovementioned literature evidence, patients who had longer hospitalization or who underwent invasive care were expected to show poor psychological outcomes.

\section{Materials and Methods}

\section{Study design}

This cross-sectional study focused on the first timepoint of a larger and longitudinal follow-up screening program aimed at assessing the psychological sequelae of individuals who were hospitalized and recovered from COVID-19, with multiple timepoints within 2 months, at 6 months, and 12 months after the hospital discharge.

\section{Participants and data collection}

Participants were recruited at the Azienda Socio-Sanitaria Territoriale Santi Paolo and Carlo (ASST-SSPC), 
a large public healthcare organization in Milan, Lombardy, who admitted 1405 COVID-19 patients during the first wave of COVID-19 pandemic (from $21^{\text {st }} \mathrm{Feb}$ ruary to $31^{\text {st }} \mathrm{July}$ ).

All recovered COVID-19 patients (defined by clinical recovery and virological clearance; see Tomasoni et al., 2020 for criteria) who had been previously hospitalized and then discharged from ASST-SSPC were contacted and offered within 2 months a multidisciplinary screening program. Data collection for the first timepoint began in early April and ended at the end of July, while data collection for the second follow-up (6 months after discharge) is currently ongoing.

\section{Measurements}

Socio-demographic characteristics (gender and age) and clinical information (days of hospitalization; the intensity of the care during hospitalization; the number of psychological sessions with the hospital psychologist after the hospitalization) were collected. Regarding the intensity of the care, patients were divided into 3 categories: i) no need of oxygen therapy or low oxygen flows; ii) subintensive care treatments with non-invasive ventilation (e.g., Continuous Positive Airway Pressure [CPAP]); iii) intensive care treatment with invasive ventilation. As far as the psychological sessions, patients who referred a need for psychological support underwent a psychological screening interview during which an eventual further psychological support of 8 sessions was offered for free, within the provisional ticket exemption for COVID-19 provided by Lombardy region. The psychologists involved in taking in charge patients recovered from COVID-19 are all psychotherapists working in the ASSTSSPC, they have different psychotherapy training backgrounds but share the expertise and the model of conducting psychological consultations for hospitalized organic patients (see Borghi et al., 2020 for details on the model of hospital psychological consultations), and receive periodically groups supervisions.

Participants were asked to complete two self-report questionnaires in order to evaluate their psychological distress after the hospital discharge for COVID-19: the Hospital Anxiety and Depression Scale (HADS) (Zigmond \& Snaith, 1983) was used to assess the prevalence of anxiety and depression symptoms while the Impact of Event Scale-Revised (IES-R) (Weiss \& Marmar, 1997) was used to evaluate the post-traumatic stress.

HADS is composed of 14 items, 7 of which evaluate the anxiety that the person is experiencing and 7 of which evaluate depression. A score in the borderline range between 8 and 11 suggests the presence of mild anxiety or depression symptoms, while a score over 11 indicates abnormal severe anxiety or depression symptoms (Bjelland, Dahl, Haug, \& Neckelmann, 2002). HADS has been validated in an Italian population and it has good psychometric properties (Iani, Lauriola \& Costantini, 2014); the validity and reliability of the Italian version of HADS are similar to those obtained with other languages (Costantini et al., 1999). Scales for anxiety and for depression showed a high internal consistency, with Cronbach's alphas ranging between 0.83 and 0.85 . Correlations between HADS and other questionnaires measuring anxiety and depression were in the range of 0.49 to 0.83 (Bjelland et al., 2002).

IES-R is composed of 22 items and it aims to evaluate the impact of a stressful event through the analysis of intrusion, avoidance and hyperarousal symptoms. IES-R has been validated in the Italian population showing good psychometric properties (Craparo, Faraci, Rotondo \& Gori, 2013), with acceptable internal consistency (Cronbach's $\alpha$ ) for each subscale (intrusion=0.87-0.94, avoidance $=0.84-0.97$, hyper-arousal $=0.79-0.91$ ) A score higher than 33 has been found (Creamer, Bell \& Failla, 2003) to indicate the possibility of an ongoing acute stress disorder (ASD) or a post-traumatic stress disorder (PTSD). Given the timing of the data collection (within two months after the stressful event), PTSD was not an option but only a long-term risk, and it will be assessed in the following two timepoints at 6 and 12 months.

During the data collection, if the questionnaires' scores were altered, the patient was offered a psychological interview with the hospital psychologist and further psychological support was considered.

The study was conducted in accordance with the Declaration of Helsinki Ethical Principles and the broader longitudinal research which comprehends the present study was approved by the Ethical Committee of the University of Milan.

\section{Data analysis}

Descriptive statistics were calculated for demographic and clinical characteristics and for psychological outcomes.

In order to test associations between psychological outcomes on continuous scales and continuous/ordinal predictors (age, days of hospitalization, the intensity of care) or dichotomous predictors (gender) Pearson correlations and t-test were used respectively.

In order to test the association between the same predictors (gender, age, days of hospitalization, and intensity of care) and each of the psychological outcomes below/above the cut-offs univariate logistic regressions were used. A standard approach for model selection was followed. In the univariate analyses, a criterion of $\mathrm{P} \leq 0.10$ was used to identify candidate predictors. Then, multivariate models were fitted and a backwards selection procedure was used to eliminate those variables not significant in the multivariate model. A criterion of $\mathrm{P} \leq 0.05$ was used for determining which variables to eliminate. P-values $\leq 0.05$ were considered to be statistically significant. All statistical tests were two-sided. P values of 0.05 or less were considered statistically significant and were conducted using the Statistical Package for Social Science (SPSS) version 26 for Mac. 


\section{Results}

\section{Sample characteristics}

A total of 264 participants were enrolled, 261 of whom completed the follow-up screening. Three patients did not complete the questionnaires due to lack of time or nonunderstanding of the Italian language: their partial answers were excluded.

Socio-demographic and clinical characteristics of the sample are depicted in Table 1. Participants are mainly males, with an average age of 59 years (st.dev. $=13.3$ ). Patients were hospitalized for a mean number of 13 days (st.dev. $=9.8$ ), and $35.2 \%$ of them received intense care (27.7 sub-intensive care and 7.5 intensive care).

\section{Psychological intervention}

Nearly $14 \%(n=36)$ of participants underwent a psychological screening interview with a hospital psychologist after the discharge. Among them, 16 (6.1\%) patients decided to begin a cycle of 8 other sessions with the hospital psychologist. The most frequent contents that emerged from the psychological supporting sessions were related to the disease experience, related hospitalization and treatments, isolation in the ward, the relationship with the healthcare provider. Particularly, all the individuals who underwent the psychological support expressed difficulties in integrating both emotionally and cognitively the lived experience with their past life and the future life. Thus, the psychological sessions were aimed to foster meaning making and help patients re-establish a coherent self-narrative that integrates the experience, while also permitting their life story to move forward along new lines.

\section{Psychological outcomes}

The distribution of the scales for the three psychological outcomes, along with the descriptive data are depicted in Table 2. Overall, the three mean scores were under the cut-offs.

Regarding the prevalence of symptoms, anxiety was frequently reported $(73,28 \%)$ by participants: $38,14.6 \%$ of them showed mild anxiety symptoms and $35,13.4 \%$ of patients had an anxiety score higher than 11 , which corresponds to severe anxiety. The prevalence of depression was $16.5 \%$ : 17 patients $(6.5 \%)$ reported a borderline score, while 27 subjects (10\%) showed severe depression symptoms.

Post-traumatic stress of 184 patients was measured using the IES-R; 77 participants did not fill in the questionnaire or had missing data. Among valid data, 67

Table 1. Sample characteristics.

\begin{tabular}{|c|c|}
\hline Characteristics & $N=261$ \\
\hline $\begin{array}{l}\text { Gender n (\%) } \\
\text { Male }\end{array}$ & $178(68.2)$ \\
\hline $\begin{array}{l}\text { Age } \\
\text { Mean (SD), range }\end{array}$ & 59 (13.3), 23-90 \\
\hline $\begin{array}{l}\text { Days of hospitalization } \\
\text { Mean (SD), range }\end{array}$ & $13(9.8), 1-65$ \\
\hline $\begin{array}{l}\text { Intensity of care received, } \mathbf{n}(\%) \\
\text { No need of oxygen therapy or low oxygen flows } \\
\text { Sub-intensive care treatments } \\
\text { Intensive care treatment }\end{array}$ & $\begin{array}{l}164(64.8) \\
70(27.7) \\
19(7.5)\end{array}$ \\
\hline $\begin{array}{l}\text { Psychological screening interview, n (\%) } \\
\text { Yes }\end{array}$ & $36(13.8)$ \\
\hline $\begin{array}{l}\text { Psychological support, n (\%) } \\
\text { Yes }\end{array}$ & $16(6.1)$ \\
\hline $\begin{array}{l}\text { Number of sessions of psychological suppor } \\
\text { Median (IQR) }\end{array}$ & $5(1-8)$ \\
\hline
\end{tabular}

Table 2. Distributions of the scales for the psychological outcomes.

\begin{tabular}{lcccccccccc}
\hline & & & & & & & \multicolumn{2}{c}{ Skewness } & \multicolumn{2}{c}{ Kurtosis } \\
\cline { 7 - 12 } & N & Range & Min & Max & Mean & St. Dev. & Statistic & St. err. & Statistic & St. err. \\
\hline HADS-Anxiety & 261 & 18 & 0 & 18 & 5.23 & 4.587 & 0.97 & 0.15 & 0.20 & 0.30 \\
\hline HADS-Depression & 261 & 15 & 0 & 15 & 3.72 & 3.497 & 1.02 & 0.15 & 0.25 & 0.30 \\
\hline IES-R & 184 & 76 & 0 & 76 & 27.13 & 21.364 & 0.61 & 0.18 & -0.77 & 0.36 \\
\hline
\end{tabular}


(36.4\%) of patients obtained a score higher than the 33, that suggests a preliminary diagnosis of PTSD.

Regarding the correlations between psychological outcomes on continuous scales and predictors, the results are depicted in Tables 3 and 4. In particular, age was found to be inversely but weakly correlate with anxiety, where younger patients displayed more severe anxiety symptoms. Contrary to our hypotheses, no correlation emerged between the length of hospitalization or the intensity of care and the psychological outcomes. Female gender was found to be significantly associated with anxiety, depression, and post-traumatic stress when compared to the male gender.

Table 5 showed data on the logistic univariate regressions for the three psychological outcomes considered below/above the cut-offs. Because only gender was found to be associated with the psychological outcomes, no multivariate regression was run. Females had a more than triple/quadruple odds of displaying anxiety, depression, and post-traumatic stress symptoms than males.

\section{Discussion}

This study assessed the psychological sequelae of recovered individuals who had been hospitalized for COVID19, within two months from the clinical and virological recovery. The aim was to explore the prevalence of distress among recovered patients, focusing on anxiety and depres- sion symptoms as well as post-traumatic stress. We found a high prevalence of psychological distress among recovered individuals. Specifically, within 2 months from hospital discharge, $28 \%$ of them showed anxiety symptoms, $16 \%$ depression symptoms, and over $36 \%$ post-traumatic stress. To our knowledge, these are the first findings exploring the psychological outcomes of being hospitalized for COVID-19, and they are quietly consistent with the previous literature on other Coronavirus, despite a lower prevalence of depression. For example, in patients recovered from SARS three months after hospital discharge the incidence of PTSD was $42 \%$, the rates of anxiety and depression were $33 \%$ and $27 \%$, respectively (Kwek et al., 2006). Similar high prevalence of anxiety, depression, posttraumatic stress, were found in the general population in Italy (Franceschini et al., 2020; Lenzo et al., 2020; Mazza et al., 2020; Rossi et al., 2020). For example, Lenzo and colleagues (2020) reported an overall prevalence of moderate-to-extremely severe anxiety, depression, and stress among general population during the lockdown $(24.4 \%$, $32 \%$, and $31.7 \%$ respectively). Preliminary studies on patients diagnosed with COVID-19 highlighted their risk of developing psychopathologies (Tomasoni et al., 2020; Bo et al., 2020), as they may have experienced the disease and the hospitalization as traumatic events.

Post-traumatic stress is linked to the individual's perception of his/her resources as not enough to cope with challenging circumstances, which therefore becomes a

Table 3. Correlations between predictors and psychological outcomes on continuos scales.

\begin{tabular}{lcccc}
\hline & $\begin{array}{c}\text { Anxiety } \\
\text { symptoms }\end{array}$ & $\begin{array}{c}\text { Depression } \\
\text { symptoms }\end{array}$ & $\begin{array}{c}\text { Post-traumatic } \\
\text { stress symptoms }\end{array}$ & $\begin{array}{c}\text { Age } \\
\text { Days of } \\
\text { hospitalization } \\
\text { of care }\end{array}$ \\
\hline Anxiety symptoms & 1 & 1 & \\
\hline Depression symptoms & $0.71^{* *}$ & $0.62^{* *}$ & 1 & 1 \\
\hline Post-traumatic stress symptoms & $0.77^{* *}$ & 0.01 & -0.11 & $0.26^{* *}$ \\
\hline Age & $-0.12^{*}$ & 0.09 & 0.10 & 1 \\
\hline Days of hospitalization & -0.04 & -0.06 & 0.01 & $0.13^{*}$
\end{tabular}

$* \mathrm{P}<0.05 ; * \mathrm{P}<0.01 .{ }^{\circ}$ Here intensity of care was condidered in its ordinal rank of three categoris: i) no need of oxygen therapy or low oxygen flows; ii) sub-intensive care; iii) intensive care treatment.

Table 4. Association between gender and psychological outcomes on continuos scales using t-test.

\begin{tabular}{|c|c|c|c|c|c|c|}
\hline Gender & Mean (st.dev) & Mean st. error & t (gl) & Mean Difference & St. Error & C.I. $95 \%$ \\
\hline \multicolumn{7}{|c|}{ Anxiety symptoms } \\
\hline Male & $4.23(3.81)$ & 0.29 & $-5.73(256)$ & -3.35 & 0.58 & $-4.50--2.20$ \\
\hline Female & $7.58(5.33)$ & 0.59 & & & & \\
\hline \multicolumn{7}{|c|}{ Depression symptoms } \\
\hline Male & $3.01(3.03)$ & 0.23 & $-5.37(256)$ & -32.40 & 0.45 & $-3.28--2.52$ \\
\hline Female & $5.41(3.90)$ & 0.47 & & & & \\
\hline \multicolumn{7}{|c|}{ Post-traumatic stress symptoms } \\
\hline Male & $22.82(18.76)$ & 1.66 & $-4.30(179)$ & -15.61 & 3.63 & $-22.850--8.38$ \\
\hline Female & $38.43(23.55)$ & 3.23 & & & & \\
\hline
\end{tabular}


traumatic experience (Lazarus \& Folkman, 1984). Individuals who were hospitalized for COVID-19 had to face multiple sources of stress: lack of information about the virus, isolation in hospital and fear for their safety and their lives. These results are consistent with those of the studies on previous epidemics, such as SARS. For instance, Kwek and colleagues (2006) reported that SARS patients continued to be functionally sub-optimal even after their discharge. Authors highlighted the difference between disease and concept illness: although the clinical episode of the infection ended, patients continued to be influenced by the past experience of the disease. In fact, SARS had a long-term impact: $44 \%$ of the sample met the diagnostic criteria for PTSD in the four years after the treatment, with a significant compromise of daily life (Hong et al., 2009).

Female gender and young age seem to be risk factors for psychological impairment, despite the finding on age is weak and inconsistent across analyses. Female patients presented higher rates of anxiety, depression and posttraumatic stress. This finding is not surprising when we take into account findings from the literature on community samples and it is consistent with the scientific literature on gender differences in psychopathology, that showed a greater depression and anxiety in women compared to men (Eaton et al., 2012; Nolen-Hoeksema, 2012). Previous studies have found that females had higher scores than males on depression, anxiety, and stress (Crawford \& Henry, 2003; Norton, 2007), even though there was no consistency across the studies (Bottesi et al., 2015). Regarding the Italian general population, some studies highlighted that women are twice as likely as men to present a mood disorder and four times more likely than men to report anxiety (Faravelli et al., 2004; De Girolamo et al., 2006). However, it is worthwhile to highlight that a significant role of gender in predicting distress during the COVID-19 outbreak was found across countries, including Italy (Lenzo et al., 2020; Mazza et al., 2020; Wang et al., 2020).

In our sample, contrary to our hypothesis, younger patients were found to show more likely anxiety symptoms, but not depression or stress. However, the correlation was weak and the association disappeared in the univariate logistic regression, calling for replications. Despite in previous works on hospitalized patients, older age was found to be associated with worse mental health outcomes (Wilkowska-Chmielewska, Szelenberger, \& Wojnar, 2013), analogous issues have been described in the literature when considering younger age in relation to virus outbreaks. Referring to past outbreaks, a worse psychological impact was associated with younger age (Brooks et al., 2020), as younger people were found to be particularly vulnerable and cope less well with the consequences of an epidemic (Taylor, Agho, Stevens \& Raphael, 2008). Similar findings were found in the general population in Italy (Lenzo et al., 2020; Mazza et al., 2020). It could be hypothesized that younger patients may think of themselves as invulnerable and did not expect the disease, with a consequent shock and a severe impact on anxiety symptoms. The next follow up at 6 and 12 months will assess whether younger patients continue to be more vulnerable than older patients or whether anxiety symptoms are an acute response to the unexpected event.

Surprisingly, no significant correlations were found between the duration of hospitalization, which is usually a measure of the severity of the disease, or the intensity of care and psychological outcomes. The finding is new and calls for replication, due to the fact that goes in the opposite direction on the previous pre-COVID literature, in which the admission in ICU and the type of treatment were found to have a significant influence on psychological distress (Kwek et al., 2006; Davydow et al., 2008).

Table 5. Predictors of psychological distress.

\begin{tabular}{|c|c|c|c|c|}
\hline & \multicolumn{4}{|c|}{ Univariate logistic regressions } \\
\hline & B (S.E.) & Wald & $\operatorname{Exp}(B)$ & $\operatorname{Exp}(B) 95 \%$ C.I. \\
\hline \multicolumn{5}{|c|}{ Anxiety symptoms (HADS-A>8 scores) } \\
\hline Gender & $1.22(0.29)$ & $17.55^{*}$ & 3.39 & $1.92-6.02$ \\
\hline Age & $-0.010(0.10)$ & 0.92 & 0.99 & $0.97-1.01$ \\
\hline Days of hospitalization & $-0.01(0.01)$ & 0.16 & 0.99 & $0.97-1.02$ \\
\hline Intensity of care ${ }^{\circ}$ & $-0.38(0.30)$ & 1.59 & 0.68 & $0.38-1.24$ \\
\hline \multicolumn{5}{|c|}{ Depression symptoms (HADS-D>8 scores) } \\
\hline Gender & $1.22(0.34)$ & $12.84 *$ & 3.39 & $1.74-6.60$ \\
\hline Age & $-0.07(0.13)$ & 0.32 & 1.01 & $0.98-1.032$ \\
\hline Days of hospitalization & $0.01(0.01)$ & 0.16 & 1.01 & $0.98-1.04$ \\
\hline Intensity of care ${ }^{\circ}$ & $-0.40(0.37)$ & 1.91 & 0.67 & $0.32-1.38$ \\
\hline \multicolumn{5}{|c|}{ Post-traumatic stress symptoms (IES-R > 33 scores) } \\
\hline Gender & $1.52(0.35)$ & $19.14^{*}$ & 4.56 & $2.31-9.00$ \\
\hline Age & $-0.01(0.01)$ & 0.49 & 0.99 & $0.97-1.01$ \\
\hline Days of hospitalization & $0.01(0.01)$ & 0.07 & 1.01 & $0.97-1.04$ \\
\hline Intensity of care $^{\circ}$ & $-0.02(0.32)$ & 0.01 & 0.98 & $0.52-1.84$ \\
\hline
\end{tabular}

* $\mathrm{P}<0.001 .{ }^{\circ}$ Because of the low number of patients receiving intensive care when comparing to the other two groups, we chose to consider the variable as dichotomous for logistic regressions. The two categories are: i) no need of oxygen therapy or low oxygen flows versus ii) sub-intensive care or intensive care treatment. 
It is also possible that patients who faced a more intensive care develop a post-traumatic growth that tempered the effects of the distressing experience on wellbeing.

These findings suggest that individuals who recovered from COVID-19 faced an extremely stressful experience. Rapid implementation of psychological intervention is essential to ensure patients protection from the psychological impact of COVID-19 (Cullen, Gulati, \& Kelly, 2020). In our sample, $13 \%$ of participants attended a psychological interview with a hospital psychologist and $6 \%$ of them were taken in charge for psychotherapy support. Exploring the contents of the psychological sessions, patients seemed to express the need to elaborate the experience they had lived (i.e., COVID-19 and related hospitalization) that is felt as traumatic, because it dramatically interrupts one's own sense of coherence (McCann \& Pearlman, 1990). Through the re-narration of the event, the psychological interview gave space and welcomed to the emotions, legitimizing the intensity of the experience. The aim was to heal the fracture between before and after the event. For many patients the return home has been challenging and has brought the difficulty of reliving daily life with a perception of normality that was still strongly compromised. Since patients have been taken in charge, their needs have been adequately addressed; therefore, we hypothesize that their prevalence of anxiety, depression and post-traumatic stress will be lower. Once longitudinal timepoints measures will be obtained, the role of early psychological support on the patients' psychological condition will be explored.

Certainly, other important factors should be taken into account to explain findings and to set up more tailored psychological interventions. Among others, the psychological vulnerability (i.e., personality variables and previous psychiatric conditions) and the infection or death of loved ones might increase the risk for developing a psychological distress response. These aspects were only clinically assessed by the psychologists during the sessions and data for all participants are not available. Future studies should carefully take into account these risk factors and assess their relevance for the patients' psychological distress. Examining the role that a complex panel of socio-demographic, clinical and premorbid factors has for the development and maintenance of depression, anxiety, and stress is important to detect people at risk of psychological disorders and to design effective and timely psychological interventions.

To the best of our knowledge, this is the first contribution examining the psychological sequelae of COVID19 among recovered individuals who were hospitalized. The further follow-up will provide a comprehensive overview and the progression of their psychological condition, with the present timepoint within two months exploring the acute psychological distress and the other two timepoints at 6 and 12 months exploring the progression of anxiety and depression symptoms, as well as the onset of PTSD.

This study has some limitations. Firstly, the data presented are cross-sectional, thus preventing from drawing causal conclusions. It is difficult to relate the high prevalence of psychological distress among recovered patients after hospitalization only by to COVID-19 and to the hospitalization, and a comprehensive and more complex assessment on precedent psychological vulnerability or resilience along with concurrent stress and risk (e.g., the death of a loved ones) factors should be assumed. However, once the research will be completed with the 6- and 12-months follow-up, the course of psychological sequelae of COVID-19 and hospitalization in recovered individuals might be better explored and understood. For example, the effect of the length of hospitalization might be explored separately for intensive/sub-intensive/nonintensive inpatients, along with their short- or long-term effects on psychological distress. Similarly, as we collected data on psychological consultation during hospitalization and on psychological support after the recovery, their preventive or moderation effect on psychological sequelae might be investigated. Moreover, the data are related to a monocentric hospital setting in Lombardy, Italy, and they call for replication in order to account for generalizability.

\section{Conclusions}

This contribution helps in understanding the mental health of recovered individuals who had been hospitalized for COVID-19. Few months after the hospital discharge, the prevalence of anxiety and post-traumatic stress were still high among patients. Female patients seem to be more vulnerable than others. Surprisingly, the day of hospitalization and the intensity of care seem not to be associated with psychological outcomes. Findings suggest the importance of a comprehensive assessment in order to set up tailored psychological interventions to support patients' mental health and to prevent long-term impact on psychological wellbeing.

\section{References}

Ahmed, H., Patel, K., Greenwood, D. C., Halpin, S., Lewthwaite, P., Salawu, A., Eyre, L., Breen, A., O'Connor, R., Jones, A., \& Sivan, M. (2020). Long-term clinical outcomes in survivors of severe acute respiratory syndrome and Middle East respiratory syndrome coronavirus outbreaks after hospitalization or ICU admission: a systematic review and meta-analysis. Journal of Rehabilitation Medicine, 52(5), 111. doi: 10.2340/16501977-2694.

Balachandar, V., Mahalaxmi, I., Subramaniam, M., Kaavya, J., Senthil Kumar, N., Laldinmawii, G., Narayanasamy, A., Janardhana Kumar Reddy, P., Sivaprakash, P., Kanchana, S., Vivekanandhan, G., \& Cho, S. G. (2020). Follow-up studies 
in COVID-19 recovered patients-is it mandatory?. Science of the Total Environment, 729, 1-8. doi: 10.1016/j.scitotenv.2020.139021

Bansal, M. (2020). Cardiovascular disease and COVID-19. Diabetes \& Metabolic Syndrome: Clinical Research \& Reviews, 14, 247-250. doi: 10.1161/CIRCULATIONAHA. 120.046941 .

Batawi, S., Tarazan, N., Al-Raddadi, R., Al Qasim, E., Sindi, A., Al Johni, S., Al-Hameed, F. M., Arabi, Y. M., Uyeki, T. M., \& Alraddadi, B. M. (2019). Quality of life reported by survivors after hospitalization for Middle East respiratory syndrome (MERS). Health and Quality of Life Outcomes, 17(1), 1-7. doi: 10.1186/s12955-019-1165-2.

Bhopal, S. S., \& Bhopal, R. (2020). Sex differential in COVID19 mortality varies markedly by age. The Lancet, 396, 532533. doi: 10.1016/S0140-6736(20)31748-7

Bjelland, I., Dahl, A. A., Haug, T. T., \& Neckelmann, D. (2002). The validity of the Hospital Anxiety and Depression Scale: an updated literature review. Journal of Psychosomatic Research, 52(2), 69-77. doi: 10.1016/s0022-3999(01)00296-3.

Bo, H. X., Li, W., Yang, Y., Wang, Y., Zhang, Q., Cheung, T., Wu, X., \& Xiang, Y. T. (2020). Posttraumatic stress symptoms and attitude toward crisis mental health services among clinically stable patients with COVID-19 in China. Psychological Medicine, 1-2. doi: 10.1017/S0033291720000999.

Borghi, L., Vegni, E.A.M., Raisi, S., Cardani, E., De Stasio, M., Leone, D., Study Group VeP (2020). La visita e parere psicologico in ambito ospedaliero un modello di intervento attraverso la pratica clinica. Psicologia della Salute, 1, 55-67. doi: 10.3280/PDS2020-001005

Bose, R. J., \& McCarthy, J. R. (2020). Direct SARS-CoV-2 infection of the heart potentiates the cardiovascular sequelae of COVID-19. Drug Discovery Today, 25, 1559-1560. doi: 10.1016/j.drudis.2020.06.021.

Bottesi, G., Ghisi, M., Altoè, G., Conforti, E., Melli, G., \& Sica, C. (2015). The Italian version of the Depression Anxiety Stress Scales-21: Factor structure and psychometric properties on community and clinical samples. Comprehensive Psychiatry, 60, 170-181. doi: 10.1016/j.comppsych.2015. 04.005

Brooks, S. K., Webster, R. K., Smith, L. E., Woodland, L., Wessely, S., Greenberg, N., \& Rubin, G. J. (2020). The psychological impact of quarantine and how to reduce it: rapid review of the evidence. The Lancet, 395, 912-920. doi: 10.1016/S0140-6736(20)30460-8

Chua, S. E., Cheung, V., McAlonan, G. M., Cheung, C., Wong, J. W., Cheung, E. P., Chan, M. T., Wong, T. K., Choy, K. M., Chu, C. M., Lee, P. W., \& Tsang, K. W. (2004). Stress and psychological impact on SARS patients during the outbreak. The Canadian Journal of Psychiatry, 49(6), 385-390. doi: 10.1177/070674370404900607.

Craparo, G., Faraci, P., Rotondo, G., Gori, A. (2013). The Impact of Event Scale-Revised: psychometric properties of the Italian version in a sample of flood victims. Neuropsychiatric Disease and Treatment, 9, 1427-1432. doi: 10.2147/NDT. S51793

Crawford, J. R., \& Henry, J. D. (2003). The Depression Anxiety Stress Scales (DASS): Normative data and latent structure in a large non-clinical sample. British Journal of Clinical Psychology, 42(2), 111-131. doi: 10.1348/014466503321 903544

Creamer, M., Bell, R., \& Failla, S. (2003). Psychometric properties of the impact of event scale-revised. Behaviour Re- search and Therapy, 41(12), 1489-1496. doi:10.1016/j.brat. 2003.07.010

Costantini, M., Musso, M., Viterbori, P., Bonci, F., Del Mastro, L., Garrone, O., Venturini, M., \& Morasso, G. (1999). Detecting psychological distress in cancer patients: validity of the Italian version of the Hospital Anxiety and Depression Scale. Supportive Care in Cancer, 7(3), 121-127. doi: 10.1007/s005209900026

Cullen, W., Gulati, G., \& Kelly, B. D. (2020). Mental health in the Covid-19 pandemic. QJM: An International Journal of Medicine, 113(5), 311-312. doi: 10.1093/qjmed/hcaa110

Davydow, D. S., Gifford, J. M., Desai, S. V., Needham, D. M., \& Bienvenu, O. J. (2008). Posttraumatic stress disorder in general intensive care unit survivors: a systematic review. General Hospital Psychiatry, 30(5), 421-434. doi: 10.1016/j.genhosppsych.2008.05.006

de Girolamo, G., Polidori, G., Morosini, P., Scarpino, V., Reda, V., Serra, G., Mazzi, F., Alonso, J., Vilagut, G., Visonà, G., Falsirollo, F., Rossi, A., \& Warner, R. (2006). Prevalence of common mental disorders in Italy. Social Psychiatry and Psychiatric Epidemiology, 41(11), 853-861. doi: 10.1016/ j.genhosppsych.2008.05.006.

Eaton, N. R., Keyes, K. M., Krueger, R. F., Balsis, S., Skodol, A. E., Markon, K. E., Grant, B. F., \& Hasin, D. S. (2012). An invariant dimensional liability model of gender differences in mental disorder prevalence: evidence from a national sample. Journal of Abnormal Psychology, 121(1), 282. doi: $10.1037 / \mathrm{a} 0024780$.

Faravelli, C., Abrardi, L., Bartolozzi, D., Cecchi, C., Cosci, F., D’Adamo, D., Lo Iacono, B., Ravaldi, C., Scarpato, M. A., Truglia, E., Rosi, S. (2004). The Sesto Fiorentino Study: background, methods and preliminary results. Psychotherapy and Psychosomatics, 73(4), 216-225. doi: 10.1159/ 000077740

Fiani, B., Covarrubias, C., Desai, A., Sekhon, M., \& Jarrah, R. (2020). A contemporary review of neurological sequelae of COVID-19. Frontiers in Neurology, 11, 640. doi: 10.3389/ fneur.2020.00640

Franceschini, C., Musetti, A., Zenesini, C., Palagini, L., Pelosi, A., Quattropani, M. C., Lenzo, V., Freda, M. F., Lemmo, D., Vegni, E., Borghi, L., Saita, E., Cattivelli, R., De Gennaro, L., Plazzi, G., Riemann, D., Castelnuovo, G. (2020). Poor quality of sleep and its consequences on mental health during COVID-19 lockdown in Italy. Frontiers, in press.

Hong, X., Currier, G. W., Zhao, X., Jiang, Y., Zhou, W., \& Wei, J. (2009). Posttraumatic stress disorder in convalescent severe acute respiratory syndrome patients: a 4-year followup study. General Hospital Psychiatry, 31(6), 546-554. doi: 10.1016/j.genhosppsych.2009.06.008.

Iani, L., Lauriola, M., \& Costantini, M. (2014). A confirmatory bifactor analysis of the hospital anxiety and depression scale in an Italian community sample. Health Qual Life Outcomes, 12(84), 1-8. doi: 10.1186/1477-7525-12-84

James, P. B., Wardle, J., Steel, A., \& Adams, J. (2020). An assessment of Ebola-related stigma and its association with informal healthcare utilisation among Ebola survivors in Sierra Leone: a cross-sectional study. BMC Public Health, 20(1), 1-12. doi: 10.1186/s12889-020-8279-7.

Ji, D., Ji, Y. J., Duan, X. Z., Li, W. G., Sun, Z. Q., Song, X. A., Meng, Y. H., Tang, H. M., Chu, F., Niu, X. X., Chen, G. F., Li, J., \& Duan, H. J.. (2017). Prevalence of psychological symptoms among Ebola survivors and healthcare workers during the 2014-2015 Ebola outbreak in Sierra Leone: a 
cross-sectional study. Oncotarget, 8(8), 12784-12791. doi: 10.18632/oncotarget.14498.

Jin, J. M., Bai, P., He, W., Wu, F., Liu, X. F., Han, D. M., Shi, L., Yang, J. K. (2020). Gender differences in patients with COVID-19: Focus on severity and mortality. Frontiers in Public Health, 8, 152. doi: 10.3389/fpubh.2020.00152

Karademas, E. C., Tsagaraki, A., \& Lambrou, N. (2009). Illness acceptance, hospitalization stress and subjective health in a sample of chronic patients admitted to hospital. Journal of Health Psychology, 14(8), 1243-1250. doi: 10.1177/ 1359105309345169.

Kwek, S. K., Chew, W. M., Ong, K. C., Ng, A. W. K., Lee, L. S. U., Kaw, G., \& Leow, M. K. S. (2006). Quality of life and psychological status in survivors of severe acute respiratory syndrome at 3 months postdischarge. Journal of Psychosomatic Research, 60(5), 513-519. doi: 10.1016/j.jpsychores.2005.08.020.

Lazarus, R. S., \& Folkman, S. (1984). Stress, appraisal, and coping. New York: Springer publishing company.

Lenzo, V., Quattropani, M. C., Musetti, A., Zenesini, C., Freda, M. F., Lemmo, D., Vegni, E., Borghi, L., Plazzi, G., Castelnuovo, G., Cattivelli, R., Saita, E., Franceschini, C. (2020). Resilience contributes to low emotional impact of the COVID-19 outbreak among the general population in Italy. Frontiers, in press.

Mazza, C., Ricci, E., Biondi, S., Colasanti, M., Ferracuti, S., Napoli, C., \& Roma, P. (2020). A nationwide survey of psychological distress among italian people during the COVID19 pandemic: Immediate psychological responses and associated factors. International Journal of Environmental Research and Public Health, 17, 1-14. doi: 10.3390/ ijerph17093165.

McCann, I. L., \& Pearlman, L. A. (1990). Psychological trauma and the adult survivor: Theory, therapy, and transformation (No. 21). New York: Psychology Press.

Ministero della salute (2020, July 31). COVID.19 - Situazione in Italia. Available from: http://www.salute.gov.it/ portale/nuovocoronavirus/dettaglioContenutiNuovoCoronavirus.jsp?lingua $=$ italiano\&id $=5351 \&$ area $=$ nuovoCoronavirus\&menu=vuoto $\% 20$ -

Nolen-Hoeksema, S. (2012). Emotion regulation and psychopathology: The role of gender. Annual Review of Clinical Psychology, 8, 161-187. doi: 10.1146/annurev-clinpsy032814-112739.

Norton, P.J. (2007). Depression Anxiety and Stress Scales (DASS-21): Psychometric analysis across four racial groups. Anxiety, stress, and coping, 20(3), 253-265.

Remuzzi, A., \& Remuzzi, G. (2020). COVID-19 and Italy: what next?. The Lancet, 395, 1225-28. doi: 10.1016/S01406736(20)30627-9.

Rogers, J. P., Chesney, E., Oliver, D., Pollak, T. A., McGuire, P., Fusar-Poli, P., ..., David, A. S. (2020). Psychiatric and neuropsychiatric presentations associated with severe coronavirus infections: a systematic review and meta-analysis with comparison to the COVID-19 pandemic. The Lancet Psychiatry, 7, 611-627. doi: 10.1016/S2215-0366(20)30203-0.
Rossi, R., Socci, V., Talevi, D., Mensi, S., Niolu, C., Pacitti, F., ..., Di Marco, A., Rossi, A., Siracusano, L., Di Lorenzo, G. (2020). COVID-19 pandemic and lockdown measures impact on mental health among the general population in Italy. Frontiers in Psychiatry, 11, 1-6. doi: 10.3389/fpsyt.2020. 00790.

Tadolini, M., Codecasa, L. R., García-García, J. M., Blanc, F. X., Borisov, S., Alffenaar, J. W., Andréjak, C., Bachez, P., Bart, P. A., Belilovski, E., Cardoso-Landivar, J., Centis, R., D’Ambrosio, L., Luiza De Souza-Galvão, M., DominguezCastellano, A., Dourmane, S., Fréchet Jachym, M., Froissart, A., Giacomet, V., Goletti, D., ... Migliori, G. B. (2020). Active tuberculosis, sequelae and COVID-19 co-infection: first cohort of 49 cases. European Respiratory Journal, 56(1). doi: 10.1183/13993003.01398-2020

Taylor, M. R., Agho, K. E., Stevens, G. J., \& Raphael, B. (2008). Factors influencing psychological distress during a disease epidemic: data from Australia's first outbreak of equine influenza. BMC Public Health, 8(1), 347. doi: 10.1186/14712458-8-347.

Troyer, E. A., Kohn, J. N., \& Hong, S. (2020). Are we facing a crashing wave of neuropsychiatric sequelae of COVID-19? Neuropsychiatric symptoms and potential immunologic mechanisms. Brain, Behavior, And Immunity, 87, 34-39. doi: 10.1016/j.bbi.2020.04.027

Tomasoni, D., Bai, F., Castoldi, R., Barbanotti, D., Falcinella, C., Mulè, Mondatore, D., Tavelli, A., Vegni, E., Marchetti, G., \& d'Arminio Monforte, A. (2020). Anxiety and depression symptoms after virological clearance of COVID-19: a cross-sectional study in Milan, Italy. Journal of Medical Virology, 1-5. doi: 10.1002/jmv.26459.

Wang, C., Pan, R., Wan, X., Tan, Y., Xu, L., Ho, C. S., \& Ho, R. C. (2020). Immediate psychological responses and associated factors during the initial stage of the 2019 coronavirus disease (COVID-19) epidemic among the general population in China. International Journal of Environmental Research and Public Health, 17(5), 1729. doi: 10.3390/ ijerph17051729

Weiss, D., \& Marmar, C. (1997). The Impact of Event Scale Revised. In J. Wilson \& T. Keane Assessing psychological trauma and PTSD: A handbook for practitioners. New York: Guilford Press.

Wilkowska-Chmielewska, J., Szelenberger, W., \& Wojnar, M. (2013). Age-dependent symptomatology of depression in hospitalized patients and its implications for DSM-5. Journal of Affective Disorders, 150(1), 142-145. doi: 10.1016/ j.jad.2012.12.012.

World Health Organization. (2020a). Coronavirus disease (COVID-19). Situation report. (August 1). Available from: https:/www.who.int/docs/default-source/coronaviruse/situation-reports/20200731-covid-19-sitrep-193.pdf?sfvrsn=42a 0221d 4

Zigmond, A. S., \& Snaith, R. P. (1983). The hospital anxiety and depression scale. Acta Psychiatrica Scandinavica, 67(6), 361-370. doi: 10.1111/j.1600-0447.1983.tb09716.x 